\title{
Universiteit
}

Leiden

The Netherlands

\section{Measuring the spring constant of a single polymer chain}

Jensenius, T.M.H.; Zocchi, G.

\section{Citation}

Jensenius, T. M. H., \& Zocchi, G. (1997). Measuring the spring constant of a single polymer chain. Physical Review Letters, 79(25), 5030-5033. doi:10.1103/PhysRevLett.79.5030

Version: Publisher's Version

License: $\quad$ Leiden University Non-exclusive license

Downloaded from: https://hdl.handle.net/1887/3213789

Note: To cite this publication please use the final published version (if applicable). 


\title{
Measuring the Spring Constant of a Single Polymer Chain
}

\author{
H. Jensenius and G. Zocchi \\ Center for Chaos and Turbulence Studies, Niels Bohr Institute, Blegdamsvej 17, 2100 Copenhagen $\emptyset$, Denmark
}

(Received 8 July 1997)

\begin{abstract}
We present a measurement of the mechanical properties of single polystyrene molecules of size $R \approx 50 \mathrm{~nm}$. Using a micromechanical technique, we show directly that the polymer chain behaves like a spring, and we measure the spring constant. We examine both cross-linked and non-cross-linked polystyrene. The former forms a stiffer spring, and the value of the spring constant is in agreement with theory, while in the latter case the measured value is larger than expected. [S0031-9007(97)04848-5]
\end{abstract}

PACS numbers: $61.41 .+\mathrm{e}$

The center of mass of a molecule is solution performs a random walk. But can the intramolecular degrees of freedom also realize a random walk? A long, flexible polymer molecule does just that, with some complications. The walk is self-avoiding, which means that interactions between monomers far apart along the chain are important; this gives rise to an excluded volume per monomer which depends on the quality of the solvent, and so on. The statics of polymer conformation is, therefore, far from trivial, but many important results can be established in terms of simple scaling arguments [1]. The dynamics is more difficult, and the mechanism of such effects as the drag reduction caused by polymers in solution is still being debated [2]. Experimentally, a direct way to probe the relaxation modes of polymer chains is in principle provided by dynamic light scattering, which measures a time dependent correlation function. Such experiments are, however, not easy to perform in the dilute regime (noninteracting coils) because the signals involved are weak, and they also require very long polymer chains. As a result, the information available on the dynamics is limited, and one often relies on more indirect methods, based on the rheological properties [3-5]. Another situation which is studied intensively is that of polymers attached to a surface. Careful mechanical measurements have been performed [6-8] which directly probe the interaction between polymer bearing surfaces, and, thus, the conformational properties of the chains. Recently, an experiment combining dynamic light scattering with the evanescent wave technique has yielded information on the dynamics of a polymer "brush" [9].

Some aspects of the dynamics of an isolated chain can be discussed in quite simple terms. For instance, if one is interested only in the longest wavelength properties ( $\lambda \sim R$, where $R$ is the size of the coil), then the polymer behaves essentially like a (overdamped) spring. It is characterized by a spring constant $K$ and a friction coefficient $\chi$, and, thus, by a relaxation time $\tau=\chi / K$ [1]. As mentioned before, direct measurements of $\tau$ are feasible but difficult. Alternatively, one can think of determining $\chi$ and $K$ separately. In this Letter, we present a direct measurement of the spring constant $K$ of a single polystyrene coil of size $R \approx 50 \mathrm{~nm}$.

Our technique is mechanical, and works as follows. A micron size sphere in water is tethered to a solid surface (glass plate) by a polymer chain. The sphere, thus, performs a confined Brownian motion. The potential which confines the sphere is determined by measuring the probability distribution of the excursions of the sphere away from its average distance from the plate. We find that the potential is a parabola, so the molecule linking the sphere to the surface behaves like a spring, and we can measure the spring constant.

While particles tethered to surfaces by polymer chains have been studied before [10-12], the length of the tether was in those cases 1 to 2 orders of magnitude larger; here we probe the mechanical properties at the usual molecular scale of nanometers. We now describe our measurements.

A dilute suspension of polystyrene spheres (diameter $10 \mu \mathrm{m})$ is placed in a cell made from a microscope slide and cover slip separated by $50 \mu \mathrm{m}$ thick spacers. The spheres settle close to the bottom plate, where they perform a vertical Brownian motion. The average separation from the plate is given by the balance between the repulsive electrostatic force (both the sphere and the plate are negatively charged) and the attractive van der Waals interaction. Thus, even for a sphere which is not tethered to the plate, the vertical motion is confined by the interaction potential with the plate. The lateral motion is, on the contrary, free Brownian motion. The experiment is based on monitoring and separation between the sphere under investigation and the plate in the course of time employing a near field optical technique which offers subnanometer resolution [13-15]. A laser beam $(20 \mathrm{~mW}$ $\mathrm{He}-\mathrm{Ne}$ laser) is brought to be totally reflected at the glasswater interface which is the bottom of the cell; on the water side of the interface no light is transmitted, but an evanescent field penetrates into the water. When a sphere is present, it scatters some of the light, the scattered intensity being a measure of the distance between the sphere and the plate, following the relation $I_{\mathrm{sc}} \propto e^{-z / \delta}$, where $z$ is the separation between the sphere and the plate $\delta$ is the 
penetration depth of the evanescent wave, which depends on the wavelength of the light, the indices of refraction, and the angle of incidence; in our case, $\delta \approx 100 \mathrm{~nm}$. The scattered light is collected by a microscope objective and focused onto a photodiode, and the intensity measured with a lock-in detection scheme. Time series of the intensity are stored on a computer for later analysis.

Intensities are converted to heights using the above relation for $I_{\mathrm{sc}}$, and a histogram of the height of the sphere is computed. The probability distribution of the height, $p(z)$, is then used to obtain the potential seen by the sphere according to $p(z) \propto e^{-\phi(z) / k T}$ where $\phi$ is the potential. Figure 1 (squares) shows one such potential, for a $10 \mu \mathrm{m}$ sphere in a $5 \mathrm{mM} \mathrm{NaCl}$ solution; the experimental points agree well with what is expected from the sum of the electrostatic and van der Waals interactions [Derjaguin-Landau-Verweg-Overbeek (DLVO) potential; dotted curve] [16]. In the course of mapping out such potentials, we observed that occasionally the sphere gets into a different state, where it is confined by a stronger force. In the experiment, this is readily seen from the size of the intensity fluctuations, which are abruptly reduced. The time series in Fig. 2 displays this transition. It is known that latex spheres may have polystyrene chains dangling from the surface [17], and we interpret the stiffer potential confining the sphere as due to one such chain adsorbing on the plate, thereby connecting the sphere to it. This tethering effect is never seen with glass beads, and working on a molecularly smooth surface (freshly cleaved mica) does not change the behavior of the polystyrene spheres. It is probable that the tether consists of a single chain because the event is rare: The spheres tumbles around in its thermal motion and only occasionally does it get trapped; it may detach again after some time. If we calculate the potential using the part of the time series which corresponds to the tethered sphere, we obtain the

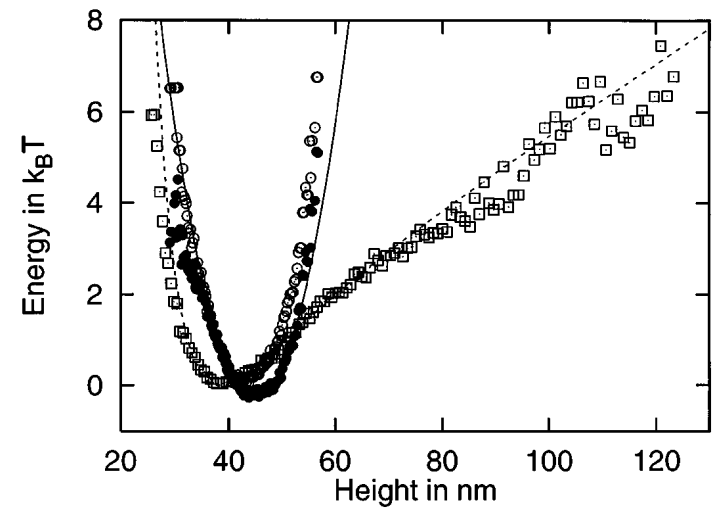

FIG. 1. Measured potential for a $10 \mu \mathrm{m}$ polystyrene sphere (non-cross-linked) in $5 \mathrm{mM} \mathrm{NaCl}$ over a glass surface: without tether (squares), with tether (open circles), and subtracting the former from the latter (full circles). Dotted line: theoretical DLVO potential (sum of electrostatic and van der Waals interactions) for this case. The continuous line is a parabola. result shown in Fig. 1 (open circles). To obtain the potential due to the tether alone, we further subtract the potential measured in the absence of the tether (squares), and obtain a parabola (Fig. 1, full circles, and Fig. 3). This is a direct demonstration that a polymer chain acts as a spring. We can measure the spring constant, and we do so for two different cases: One set of experiments was performed with polystyrene spheres for which the polymer chains are cross-linked ( $2 \%$ cross-linking). The tether confining these spheres is then also a $2 \%$ cross-linked polystyrene coil. In this case, the measurements give a value of the spring constant $K \approx 1.5 \times 10^{-3} \mathrm{~N} / \mathrm{m}$. Figure 3 shows one of these potentials.

A second set of experiments was performed with polystyrene spheres for which the polymer (and, thus, the tether confining the sphere) is not cross-linked. We observed qualitatively the same behavior, but we found values of the spring constant 5-10 times smaller. Thus, cross-linking changes the measured value of the spring constant substantially, and for a quantitative understanding of the data, this effect has to be taken into account. In this case (non-cross-linked polystyrene), the measured values of $K$ are comprised between $2 \times 10^{-4}$ and $3 \times 10^{-4} \mathrm{~N} / \mathrm{m}$. Figure 4 shows the potential for a non-cross-linked sphere; the corresponding spring constant is $K \approx 2.9 \times 10^{-4} \mathrm{~N} / \mathrm{m}\left(K \approx 2.4 \times 10^{-4} \mathrm{~N} / \mathrm{m}\right.$ for the case shown in Fig. 1). We note that these are soft springs: An applied force of $1 \mathrm{pN}$ would cause a $5 \mathrm{~nm}$ displacement, or $10 \%$ of the length of the spring. On a different surface (mica), we obtain the same values (both for cross-linked and non-cross-linked polystyrene).

We now interpret the measurements quantitatively. A (noncollapsed) polymer coil is an "entropic" spring: Stretching or compressing the coil away from its equilibrium size decreases the number of possible conformations and, thus, the entropy; the free energy correspondingly increases. A simple calculation shows that the free energy is quadratic in the change of chain size and that the spring

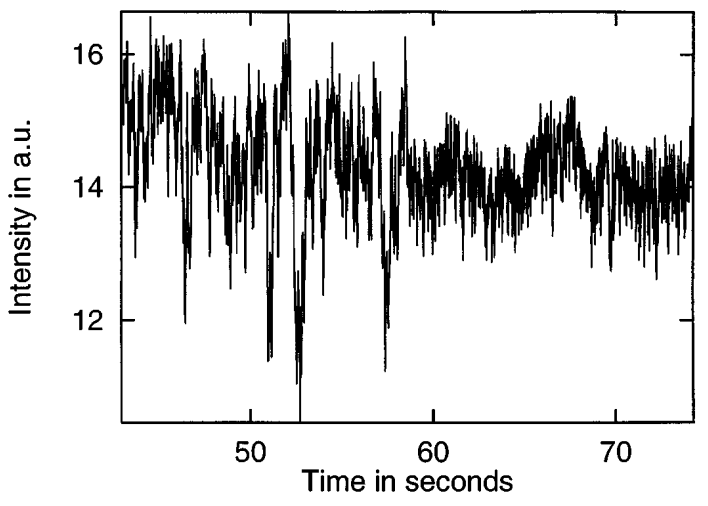

FIG. 2. Time series of the light scattered by a sphere, showing an abrupt reduction in the intensity fluctuations when the sphere gets tethered. 


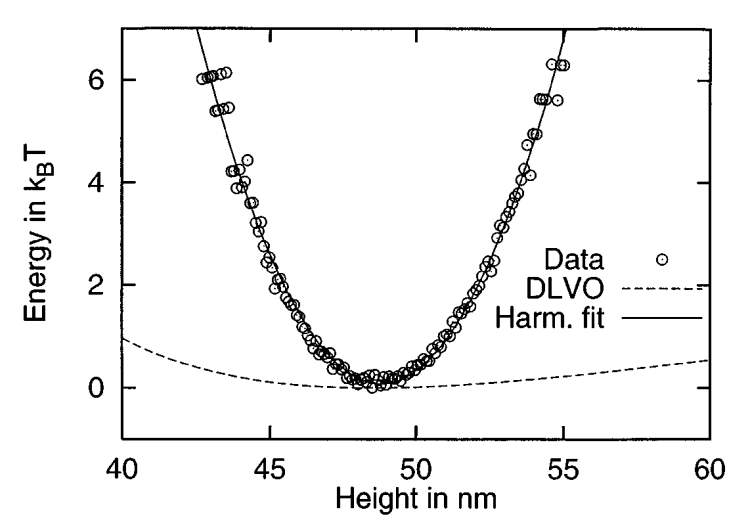

FIG. 3. The potential for a tethered sphere of cross-linked polystyrene. $3 \mathrm{mM} \mathrm{NaCl}$, glass surface. $K \approx 1.5 \times$ $10^{-3} \mathrm{~N} / \mathrm{m}$. Broken line: DLVO potential; continuous line: harmonic potential.

constant is

$$
K=\frac{3 k T}{R^{2}},
$$

where $R$ is the size of the coil, $T$ is the temperature, and $k$ is Boltzmann's constant [1]. In the experiment, $R \approx$ $50 \mathrm{~nm}$ and the above formula gives $K \approx 5 \times 10^{-6} \mathrm{~N} / \mathrm{m}$.

Let us now consider cross-linked chains; the overall size of the chain is $R$, and the coil can be though of as consisting of $n_{B}$ blobs of size $R_{B} ; n_{B}$ is the number of cross-links in the coil. The blobs are close packed:

$$
n_{B}=\left(R / R_{B}\right)^{3}
$$

and each has a spring constant

$$
K_{B}=\frac{3 k T}{R_{B}^{2}} .
$$

It is easy to show that the elastic constant $E$ (units of pressure) of such a material is

$$
E=\frac{3 k T}{R_{B}^{3}}
$$

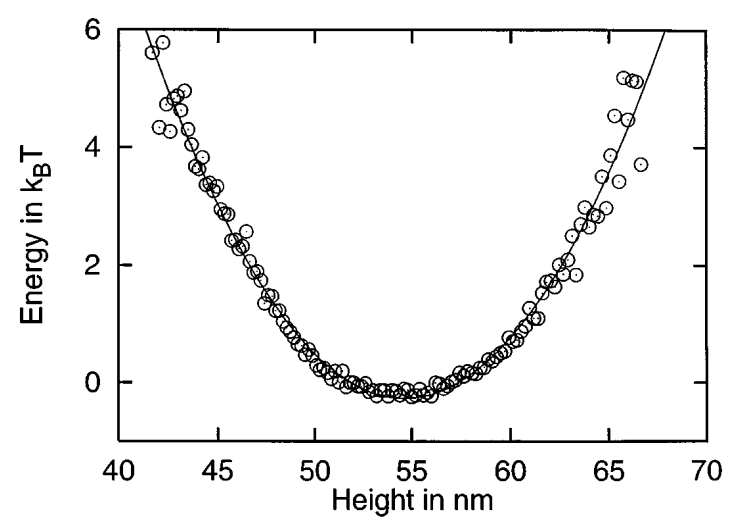

FIG. 4. The potential for a tethered sphere (non-cross-linked polystyrene) is $3 \mathrm{mM} \mathrm{NaCl}$ on mica. The length through the data is a parabola. The corresponding spring constant is $K \approx 2.9 \times 10^{-4} \mathrm{~N} / \mathrm{m}$. this formula is written $E=3 k T c / N_{B}$ in the context of polymer gels [1], where $c$ is the concentration and $N_{B}$ is the number of monomers in a blob: $c=N_{B} / R_{B}^{3}$. To compress the coil by $\Delta R$, one needs to apply a force $F=R^{2} E \Delta R / R$, i.e., the spring constant of the coil is

$$
K_{\text {cross-linked }}=\frac{3 k T}{R_{B}^{2}} \frac{R}{R_{B}} .
$$

Compared to the spring constant of a non-cross-linked coil of the same size $R$, we have:

$$
\frac{K_{\text {cross-linked }}}{K}=\left(R / R_{B}\right)^{3}=n_{B}
$$

To estimate the number of cross-links $n_{B}$, we have to know the number of monomers. We use the ideal chain relation $R=a N^{1 / 2}$, where $a$ is the persistence length ( $a \approx 1.5 \mathrm{~nm}$ for polystyrene) and $\mathrm{Na}$ is the total length of the stretched chain; the number of monomers $N_{m}$ is, for polystyrene, related to $N$ by $N_{m} \approx 10 \mathrm{~N}$. With $R \approx 50 \mathrm{~nm}$, this gives $N \approx 10^{3}$ and $N_{m} \approx 10^{4} \mathrm{~nm}$. Two percent cross-linking means $n_{B}=2 / 100 \times N_{m}=200$, and, therefore, $K_{\text {cross-linked }} \approx 200 \times 5 \times 10^{-6} \mathrm{~N} / \mathrm{m}=$ $10^{-3} \mathrm{~N} / \mathrm{m}$, which is close to the value we measure $\left(1.5 \times 10^{-3} \mathrm{~N} / \mathrm{m}\right)$. Thus, in the case of the cross-linked polymer, the measured spring constant is in quantitative agreement with expectations. This also confirms that the spheres are tethered by a single coil. Indeed, this conclusion is supported by the following facts: (1) The "tethering" event is rare; (2) measured values of $K$ for different spheres (of the same kind) are close; (3) the numerical value found is consistent with a single coil (in the case of $2 \%$ cross-linking).

We now consider the measurements on the non-crosslinked polystyrene. Here, we find values of the spring constant $K \approx 2.5 \times 10^{-4} \mathrm{~N} / \mathrm{m}$, i.e., a factor of 50 larger than the ideal chain estimate $K=3 k T / R^{2}$. This is an interesting result. It shows that the tether, in this case, is not an ideal chain. Indeed, we could account for the measured values of $K$ by introducing, even in this case, an "effective" cross-linking (a density of "permanent" entanglement points) of order $0.5 \%$. This is, however, speculative.

In conclusion, through a micromechanical experiment probing $\mathrm{nm}$ displacements and $\mathrm{pN}$ forces we show directly that a polymer chain behaves like a spring. In the case of cross-linked chains, the measured spring constant is in good agreement with what is expected from theory. For non-cross-linked chains, the measured spring constant is larger than the value for an ideal chain, possibly due to entanglement effects. Our work demonstrates that the technique which we employ can be successfully applied to the study of the mechanical properties of macromolecules.

This work was supported in part by a grant from the Danish Natural Science Research Council (Contract No. 9400374). 
[1] P. G. de Gennes, Scaling Concepts in Polymer Physics (Cornell University Press, Ithaca, 1979).

[2] W. M. Kulicke et al., Adv. Polym. Sci. 89, 1 (1989).

[3] S. A. Adelman and K. F. Freed, J. Chem. Phys. 67, 1380 (1977).

[4] K. L. Ngai and G. D. J. Phillies, J. Chem. Phys. 105, 8385 (1996).

[5] J.W.M. Noordermeer et al., Macromolecules 8, 539 (1975).

[6] J. N. Israelachvili et al., Macromolecules 17, 204 (1984).

[7] J. Klein and P.F. Luckham, Macromolecules 17, 1041 (1984).

[8] L. Dai and C. Toprakcioglu, Europhys. Lett. 16, 331 (1991).

[9] G. Fytas et al., Science 274, 2041 (1996).

[10] T. Dabros, P. Warszynski, and T.G. M. van de Ven,
J. Colloid Interface Sci. 162, 254 (1994).

[11] M. Kamiti and T. G. M. van de Ven, Macromolecules 29, 1191 (1996).

[12] P. Cluzel et al., Science 271, 792 (1996).

[13] D. C. Prieve and N. A. Frej, Langmuir 6, 396 (1990).

[14] D. C. Prieve and J. Y. Walz, Appl. Opt. 32, 1629 (1993).

[15] G. Zocchi, Europhys. Lett. 35, 633 (1996).

[16] We obtain the mean value of the absolute distance by deducing, from the same data, the diffusion constant of the sphere. The diffusion constant is reduced by the proximity of the surface, and the coefficient is given by the ratio of the distance to the wall over the radius of the sphere.

[17] E. Evans (private communication); see also E. Evans, K. Ritchie, and R. Merkel, Biophys. J. 68, 2580 (1995). 